\title{
Acoustic methods in real-time welding process monitoring: Application and future potential advancement
}

\author{
M. F. M. Yusof' ${ }^{1}$, M. Ishak' ${ }^{1}$, and M. F. Ghazali ${ }^{1}$ \\ ${ }^{1}$ Faculty of Mechanical and Automotive Engineering Technology, Universiti Malaysia Pahang, 26600 Pekan, Pahang, Malaysia \\ Phone: +6094246319; Fax: +609424222
}

\begin{abstract}
The rapid advancement of the welding technology has simultaneously increased the demand for the online monitoring system in order to control the process. Among the methods that could be possibly used to assess the weld condition, an air-borne acoustic method grasps the attention from scholars due to its ability to provide a simple, non-contact, and low-cost measurement system. However, it is still lack of resources involving this subject in an attempt to deeply understand the emitted sound behaviour during welding especially when dealing with a complete deviation of a process parameter, welding types, workpiece material as well as the noise from the surrounding. This paper reviews the application of the acoustic method in monitoring the welding process. Specifically, this review emphasized the source of both structure-borne and airborne acoustic during the welding process and the significance of applying the acoustic method in more detail. By focusing on the liquid state welding process, the scope of discussion converged on the arc and laser welding process. In the last part of this review, the potential future advancement of this method is pointed out before the overall conclusion is made.
\end{abstract}

ARTICLE HISTORY

Received: $12^{\text {th }}$ Oct. 2020

Revised: $25^{\text {th }}$ May 2021

Accepted: $05^{\text {th }}$ June 2021

\section{KEYWORDS}

Acoustic method;

real-time monitoring;

SMAW;

GMAW;

laser welding

\section{INTRODUCTION}

Welding is one of the oldest metal joining methods which have been applied in numerous cluster of industries since many decades ago. Large industries such as automotive, oil and gas and shipbuilding have employed this technology owing to its capability in promoting high strength and wide range of processes option [1,2]. Accordingly, the rapid evolution of welding technology could be observed for many years behind.

Despite the advancement of welding technologies, reaching the process stability is still quite challenging and identifying the optimum welding condition is one of the potential solutions to achieve the stability as well as suppress the number of defects. By this reason, studies associate with the process optimization were done to align with the advancement of welding technology since the past several decades [3-8]. According to Lee et. al [9], it was proven that the experimental studies for process optimization were significant in understanding the parameter effect to weld quality and reproducibility. However, it tends to be insignificant if the process parameters respond in a non-linear way due to numerous disturbance during the welding process. Moreover, other uncertainties such as fault occurring in the welding machine system, material imperfection, and unforeseen contamination would also contribute to the degradation of weld quality [1]. This is among the reasons why quality assurance still becomes a major issue in the production plan, even though the optimized parameters for the process were identified. Thus, many scholars agree that the development of inprocess monitoring system is the essential solution to this problem. This is due to its advantageous in reducing the amount of reject, improve reproducibility, save cost, and also enhance the development of the process control system in future [10-13].

In essence, physical responses in the form of electrical, thermal, optical, image, and acoustic dynamically emerged from the welding process. By this reason, monitoring the welding process is commonly done by gaining and understanding the information concerning these responses. In work related to the arc welding process, the use of current, voltage and power acquired from the process have been demonstrated to monitor the weld quality [14-18]. On the other hand, it was shown that the interpretation of plasma charge signal significantly assisted in monitoring both plasma cloud and oscillation behaviour which closely connected to penetration condition and also the presence of defects during laser welding process [19-21]. Apart from the electrical signal, other works proved that the uses of the thermal signal are also helpful in determining the welding condition. By utilizing probes such as pyrometer [22], and infrared camera [23-27], the temperature distribution in the weld pool and its trend during the cooling process could be monitored. Moreover, in the case of laser welding, the drop in laser power, presence of contamination, and also lack in shielding gas could also be detected. Besides, the use of optical signal was also proved to be significant in monitoring the welding process. In the case of the arc welding process, arc plasma that emerged during the process produce a significant amount of optical signal. Meanwhile, in the laser welding process, the optical signal produced from the laser-material interaction. Therefore, the use of spectrometer [28-31] for monitoring purpose also have been demonstrated for detecting penetration status, porosity, gap-instabilities, and local thickness reduction. On the other hand, the use of imaging method using high-speed cameras 
also proven to be significant in giving information with respect to the weld pool behaviour [32-36], the characteristic of metal droplet transfer [37-39], plasma plume [40-42] as well as the keyhole behaviour [43-47]. In another approach, several lines of evidence show that defect such as burn through [48-52], humping [53], and porosity [54-56] could be detected through the analysis of the acquired acoustic signal. Moreover, phenomena associates with metal transfer characteristic [51, 57, 58], weld pool oscillation [59], and plasma plume formation [60, 61] have also been studied using this approach.

Based on the overview, several approaches could be taken to monitor the welding process. Every of each method could provide a significant result differently. Among the various methods, acoustic method draws the attention from previous researchers owing to some of their unique features such as simple system, responsible high speed, non-contact (for the case of air-borne acoustic signal), and convenient sensor setup [49, 59, 62, 63]. On that account, the idea of the present work is to provide a comprehensive review of the application of this method in monitoring and assessing the weld quality. Several series of review has been done on research associates with monitoring studies during welding process previously [10, 13, 64]. However, authors tend to highlight various types of monitoring method in which the use of the acoustic method is just generally discussed. This review emphasized on the source of both structure-borne and air-borne acoustic during the welding process and the significance of applying the acoustic method in more detail. By focusing on the liquid state welding process, the scope of discussion converged on the arc and laser welding process. In the last part of the review, the potential future advancement of this method is pointed out before the overall conclusion is made.

\section{ACOUSTIC METHOD FOR MONITORING THE ARC WELDING PROCESS}

As underlined, the acoustic method implies either by acquiring structure-borne or air-borne acoustic wave signal. The frequency ranges of acoustic emission phenomena are typically from $20 \mathrm{kHz}$ to $3 \mathrm{MHz}$. Under this fact, the acquired signal is usually not influenced by the harsh environmental noise that arises from the process [65, 66]. Basically, this type of signal is an elastic wave produced by the rapid release of energy from the sudden redistribution of stress in the material [66], and this wave is propagating in the structure itself. Besides, owing to its nature of propagating through a solid structure, acquiring structure-borne acoustic wave is commonly done by using a piezoelectric sensor which is contacted directly on the surface of a workpiece. Meanwhile, a microphone is used to acquire the audible sound from the welding process in which it is a non-contact method because it was typically located at a certain distance from weld point [53, 63, $67,68]$.

\section{Source of Acoustic Wave in Arc Welding Process}

In the arc welding process, the structure-borne acoustic emission was found mainly originated from the change in microstructure, internal stress, and plastic deformation [50]. However, the formation of this type of wave from those phenomena is still considered as a complex problem since the lack number of research focused on physically governing the equation.

Unlike acoustic emission, the audible sound signal was mostly present from the arc ignition process, which changes its pattern from the variation of current and voltage [69]. Besides, other phenomena such as molten pool oscillation [50], keyhole formation [70] and spatter [71] were also found as the primary source of sound during the arc welding process. In GMAW process, sound could appear in impulse mode and turbulent noise [69]. The impulse mode of sound was strongly related to the rapid current change which was originated from the short-circuiting process between electrode and material, arc extinction, and partial spraying of molten metal drop when it strikes the molten pool. Accordingly, the impulse sound continues to generate by the disengagement of the molten metal from the electrode which later on creates the spark ignition and consequently cause a rapid increase on temperature and expand the shielding gas to oscillate the air in an audible frequency range [72]. On the other hand, the turbulent noise emerges from many sources, but molten oscillation is one of the critical source [73, 74]. In GTAW process, the behavior of the sound was not much different as compared to GMAW process. The obvious different was only the absence of sound source from metal droplet transfer [90]. Therefore, much of work related to GTAW process revealed the information related to the weld condition from the sound originated from the weld pool oscillation [75-77] and arc sound [56, 78].

In an earlier study [79], the correlation between acoustic sound pressure with the arc voltage supply was represented through a mathematical equation. Basically, the emerged sound pressure is assumed to be directly proportional to the time derivatives of the electrical power supply to the arc as shown by Eq.(1) whereas $C_{l}, U(t)$ and $I(t)$ are the proportionality factor, potential and current respectively.

$$
P(t)=C_{1} \frac{\partial}{\partial t}[U(t) . I(t)]
$$

Meanwhile, the proportionality factor depends on the geometrical factor, which is influenced by the distance of sound source and microphone, adiabatic expansion coefficient $K$, and speed of sound $c$ [80].

$$
C_{1}=\alpha\left(\frac{K-1}{c^{2}}\right)
$$




\section{Application of Acoustic Method for Monitoring Arc Welding Process}

Table 1 shows the summary of research associates with the application of the acoustic method for weld quality assessment during the arc welding process. It could be observed that the study of the acoustic emission method for weld quality monitoring started many decades ago. In early research by Wehrmeister [81], structure-borne acoustic emission was acquired to detect several types of defect existed from the submerged welding process. In that study, the test was done in offline mode, and the acoustic emission signal was initiated from the load given to the sample. By determining the signal features, crack and slag entrapment were successfully detected. However, the presence of porosity was only able to be detected when a crack also exists together. A similar approach also has been taken in recent research by Droubi et. al [82] whereas the acquisition process was done during offline mode. Unlike the other earlier work, other works imply the spectrum and time-frequency analysis together with the typical acoustic emission feature analysis to detect slag, crack and porosity. It was found that the application of time-frequency method called wavelet transform has improved the sensitivity of Acoustic Emission method in detecting those types of defects. Besides detecting a defect, Stephanova et. al [83], have demonstrated the use of time arrival analysis and floating threshold to detect and locate defects such as pores, cracks, slag, as well as faulty fusion.

Apart from detecting and locating the defects, studies reveal that another information contained in the acquired acoustic emission signal was related to the micro-transformation of the grain structure from heating and fusion process. For instance, Luo Yi et. al [84] have studied the correlation between the acquired structure-borne acoustic signal with the grain evolution due to the change in temperature during the arc welding process. The result from the study revealed that the increase of weld heat input caused the increasing of AE count due to the growth of grain. In another study, Takayama et. al [85] also found that the acoustic emission signal gave a piece of important information concerning the zinc embrittlement phenomena which lead to the crack formation in the fusion zone. Based on these findings, if the characteristic of the acoustic emission signal from Gas Metal Arc Welding (GMAW) and Gas Tungsten Arc Welding (GTAW) were compared, no significant difference was pointed by the previous research until now [74]. This is due to the fact that the main source of this signal was from the micro-transformation of the microstructure during melting and solidification phase.

Table 1. Summary of studies associates with the use of the acoustic emission method for monitoring and assessing the weld quality during the arc welding process

\begin{tabular}{|c|c|c|c|c|c|c|}
\hline Author & Methodology & Material & $\begin{array}{l}\text { Welding } \\
\text { Type }\end{array}$ & $\begin{array}{c}\text { Pre- } \\
\text { Processing }\end{array}$ & Analysis & $\begin{array}{l}\text { Type of Defect/ } \\
\text { phenomena }\end{array}$ \\
\hline $\begin{array}{l}\text { Wehrmeister } \\
1978 \\
{[81]}\end{array}$ & $\begin{array}{l}\text { Butt Weld } \\
\text { (submerged } \\
\text { arc weld) }\end{array}$ & & ERW & N/A & AE features & $\begin{array}{l}\text { 1. Slag entrapment } \\
\text { 2. Crack }\end{array}$ \\
\hline $\begin{array}{l}\text { Takayama et } \\
\text { al } 2012[85]\end{array}$ & N/A & $\begin{array}{l}\text { AISI } 304 \\
\text { steel }\end{array}$ & $\begin{array}{l}\text { Not } \\
\text { Described }\end{array}$ & $\begin{array}{l}\text { Wavelet } \\
\text { transform }\end{array}$ & Source Location & $\begin{array}{l}\text { Zinc Embrittlement } \\
\text { cracking }\end{array}$ \\
\hline $\begin{array}{l}\text { Stephanova } \\
\text { et. al } 2015 \\
{[83]}\end{array}$ & N/A & N/A & $\begin{array}{l}\text { Not } \\
\text { Described } \\
\text { (offline) }\end{array}$ & N/A & $\begin{array}{l}\text { 1. Time Of Arrival } \\
\text { Analysis } \\
\text { 2. Floating threshold }\end{array}$ & $\begin{array}{l}\text { Located defect such as } \\
\text { pores, cracks, slag, faulty } \\
\text { fusions, and titanium } \\
\text { inserts }\end{array}$ \\
\hline $\begin{array}{l}\text { Luo Yi et. al } \\
2016 \\
{[84]}\end{array}$ & N/A & N/A & GWAW & N/A & 1. AE Count & Grain size \\
\hline $\begin{array}{l}\text { Droubi et al } \\
2017 \\
{[82]}\end{array}$ & Butt Weld & $\begin{array}{l}\text { Carbon } \\
\text { Steel }\end{array}$ & $\begin{array}{l}\text { Not } \\
\text { Described }\end{array}$ & N/A & $\begin{array}{l}\text { 1. AE Features } \\
\text { 2. Band Energy } \\
\text { 3. Wavelet analysis }\end{array}$ & $\begin{array}{ll}\text { 1. } & \text { Slag } \\
\text { 2. } & \text { Crack } \\
\text { 3. } & \text { Porosity }\end{array}$ \\
\hline
\end{tabular}

As previously explained, metal droplet transfer was one of the main sources of the sound formation in GMAW. This fact results in thorough investigation towards the characteristic of sound from metal droplet transfer phenomena to reveal the information related to the weld quality. According to early research by Saini and Floyd [57] both time domain and frequency domain parameters have been extracted from the acquired sound in an attempt to identify the metal transfer mode during the arc welding process. In the study by Grad et al [50], the stability of the GMAW process was observed from the acquired sound signal. Moreover, the effect on carbon content to the sound signal was also being investigated. According to the findings, any non-regularity associates with the arc-ignition which formed a burn-through defect could be detected from the kurtosis value of the sound signal. In more specific, it was revealed in their study that the kurtosis value gains its value according to the increase in metal droplet transfer frequency. This frequency will determine the stability of the process, whereas the lower frequency will lead to the process instability, which later on causes the formation of the defect. Furthermore, no significant change in the sound pattern was recorded due to the change in carbon content. In another study by Kamal et. al [58], the kurtosis of the acquired sound was found to be a useful indicator to determine the metal deposition stability. It was reported that the kurtosis of the acquired sound inversely proportional to 
the deposition efficiency. Moreover, in another part of their study [51], they have found out that RMS and kurtosis of the acquired sound gave significant information with respect to the metal transfer mode.

Apart from metal droplet transfer, the characteristic of the sound from the arc ignition were also proven to be significantly related to the weld condition. Fidali et. al [86] in their work uses the Inter-quartile range (IQR) parameter to detect any instability during the arc welding process. In another similar study, Cayo et. al [87] have demonstrated the use another sound features such as sound pressure level and acoustic ignition frequency for this purpose. Besides, Kamal and Surdjya [88] revealed the correlation between the sound acquired from weld with different in the depth of penetration. In their research, the kurtosis of sound, arc power and weld peak temperature could be simultaneously measured to monitor the depth of penetration. Meanwhile, Yusof et. al [68] demonstrate the use of the air-borne acoustic method to detect the porosity underneath the weld bead during the arc welding process. In their work, the acquired signal was decomposed using Hilbert Huang transform to enhance the sensitivity of this method in detecting the porosity.

Besides detecting defects and monitoring the penetration status, the implementation of the air-borne acoustic method also involved classification of the weld quality as well as the prediction both from the numerical and experimental analysis. For instance, Sumesh et. al [52], applied machine learning method for the classification of the sound signal acquired from different weld quality. It was reported that by using the random forest algorithm, defects such as burn through and lack of fusion could be classified with the efficiency of $88.69 \%$. Unlike the other works, Horvat et. al [72] have developed the mathematical model to calculate the sound pattern from the current data. From the calculated sound signal, the stability of the welding process could be evaluated without the influence of the environmental noise which polluting the acquired sound from the experiment.

In studies related to GTAW process, the weld quality was assessed by the deep comprehend on the sound emerged from the arc ignition, keyhole formation, and molten metal oscillation. In the work by Wang et al [89], the direction was more towards gaining the understanding of how weld parameters affect the characteristic of the generated arc sound. In their study, it was revealed that parameters such as weld speed and arc length give significant influence to the characteristic of sound. Unlike the other findings, Saad et al [90] implements the neural network analysis for identifying cutting and keyhole mode during the welding process. Concurrently, Wang \& Zhao [70] introduced Asp algorithm to determine the molten pool status, which was related to the weld penetration status. This algorithm was useful in distinguishing the non-penetrated phase, the transition phase, as well as keyhole phase. Meanwhile, several lines of evidence prove that the classification efficiency could be enhanced by selecting the optimum features. As an example, hybrid Fisher-based filter and wrapper have been applied in work by Zhang et al [56] for this purpose. On the other hand, Ye et. al [91] used branch and bound method to select the sound features which represent the penetration condition. At the same time, the genetic algorithm was utilized by Wang et al [74] to improve the classification of the sound signal from different weld penetration. In another unique work, Lv et. al [78] not only predict the depth of penetration from the neural network analysis of the acquired sound but also design the controller to control the input current depending on the variation of arc sound pattern. The online penetration control was done based on the Back Propagation Artificial Neural Network (BPANN) model.

Table 2. Summary of studies associates with the use of the air-borne acoustic method for monitoring and assessing the weld quality during the arc welding process

\begin{tabular}{|c|c|c|c|c|c|c|}
\hline Author & Methodology & Material & $\begin{array}{l}\text { Welding } \\
\text { Type }\end{array}$ & $\begin{array}{c}\text { Pre- } \\
\text { Processing }\end{array}$ & Analysis & $\begin{array}{l}\text { Type of Defect/ } \\
\text { phenomena }\end{array}$ \\
\hline $\begin{array}{l}\text { Saini \& } \\
\text { Floyd } 1998 \\
{[57]}\end{array}$ & N/A & Mild Steel & GMAW & N/A & $\begin{array}{ll}\text { 1. } & \text { Pulse rate } \\
\text { 2. } & \text { Fourth root } \\
\text { Kurtosis } \\
\text { 3. Zero-crossing } \\
\text { rate } \\
\text { 4. Shape factor } \\
\text { 5. } \\
\text { 6. Impst factor } \\
\text { 7. } \\
\text { Clearance } \\
\text { factor }\end{array}$ & $\begin{array}{l}\text { Weld Transfer } \\
\text { Mode ( Short } \\
\text { Circuiting \& } \\
\text { Spray Mode) }\end{array}$ \\
\hline $\begin{array}{l}\text { Wang \& } \\
\text { Zhao } 2001 \\
{[70]}\end{array}$ & Bead on plate & $\begin{array}{l}\text { Stainless } \\
\text { steel }\end{array}$ & GTAW & STFT & $\begin{array}{l}\text { Asp algorithm, } \\
\text { Variance }\end{array}$ & $\begin{array}{l}\text { Molten pool } \\
\text { status (keyhole) }\end{array}$ \\
\hline $\begin{array}{l}\text { Grad et al } \\
2004[50]\end{array}$ & N/A & $\begin{array}{l}\text { Low carbon } \\
\text { steel (DIN } \\
\text { Rst13) \& } \\
\text { Medium } \\
\text { Carbon steel } \\
\text { (DIN CK45) }\end{array}$ & GMAW & N/A & Kurtosis & $\begin{array}{l}\text { Stability } \\
\text { (Burn Through) }\end{array}$ \\
\hline
\end{tabular}


Table 2. Summary of studies associates with the use of the air-borne acoustic method for monitoring and assessing the weld quality during the arc welding process (cont.)

\begin{tabular}{|c|c|c|c|c|c|c|}
\hline Author & Methodology & Material & $\begin{array}{c}\text { Welding } \\
\text { Type }\end{array}$ & $\begin{array}{c}\text { Pre- } \\
\text { Processing }\end{array}$ & Analysis & $\begin{array}{l}\text { Type of Defect/ } \\
\text { phenomena }\end{array}$ \\
\hline $\begin{array}{l}\text { Saad et al } \\
(2006)[90]\end{array}$ & VPPAW & Al 5256 & GTAW & N/A & $\begin{array}{l}\text { 1. Time Domain } \\
\text { 2. Frequency } \\
\text { 3. ANN }\end{array}$ & $\begin{array}{l}\text { Cutting /Keyhole } \\
\text { Mode }\end{array}$ \\
\hline $\begin{array}{l}\text { Cayo et al } \\
2009 \text { [87] }\end{array}$ & N/A & $\begin{array}{l}\text { AISI } 1020 \\
\text { Steel }\end{array}$ & GMAW & N/A & $\begin{array}{ll}\text { 1. } & \text { Acoustic } \\
\text { Ignition } \\
\text { Frequency } \\
\text { 2. } & \text { Sound } \\
\text { Pressure } \\
\text { Level }\end{array}$ & Process Stabiity \\
\hline $\begin{array}{l}\text { Wang et al } \\
2009 \text { [89] }\end{array}$ & N/A & $\begin{array}{l}\text { Alluminium } \\
\text { alloy }\end{array}$ & GTAW & N/A & $\begin{array}{l}\text { Time domain and } \\
\text { frequency domain } \\
\text { charcteristic }\end{array}$ & $\begin{array}{l}\text { Characteristic of } \\
\text { sound from } \\
\text { variation of gas } \\
\text { flow, microphone } \\
\text { angle, weld speed } \\
\text { and arc length. }\end{array}$ \\
\hline $\begin{array}{l}\text { Kamal et al } \\
2009 \text { [58] }\end{array}$ & N/A & N/A & GMAW & N/A & $\begin{array}{l}\text { 1. Kurtosis } \\
\text { 2. Fast Fourier } \\
\text { TransformT }\end{array}$ & $\begin{array}{l}\text { Metal Deposition } \\
\text { Efficiency } \\
\text { (Stability) }\end{array}$ \\
\hline $\begin{array}{l}\text { Kamal et al } \\
2010 \text { [51] }\end{array}$ & Bead on plate & Mild Steel & GMAW & N/A & $\begin{array}{l}\text { 1. Kurtosis } \\
\text { 2. RMS }\end{array}$ & $\begin{array}{l}\text { Metal transfer } \\
\text { mode, stability, } \\
\text { blow hole }\end{array}$ \\
\hline $\begin{array}{l}\text { Horvat et al } \\
2011 \text { [72] }\end{array}$ & & Steel & GMAW & N/A & $\begin{array}{ll}\text { 1. } & \text { Numerical } \\
\text { Analysis } \\
\text { 2. } & \text { Sound } \\
\text { Pressure } \\
\text { Level }\end{array}$ & \\
\hline $\begin{array}{l}\text { Kamal \& } \\
\text { Surjya } 2011 \\
{[88]}\end{array}$ & Bead on plate & $\begin{array}{l}\text { Low Carbon } \\
\text { Steel }\end{array}$ & GMAW & N/A & Kurtosis & DOP \\
\hline $\begin{array}{l}\text { Ye et al } 2011 \\
{[91]}\end{array}$ & N/A & $\begin{array}{l}\text { Alluminium } \\
\text { Alloy }\end{array}$ & GTAW & $\begin{array}{l}\text { Wavelet } \\
\text { transform }\end{array}$ & $\begin{array}{l}\text { Branch and } \\
\text { Bound (BB) } \\
\text { method. (Feature } \\
\text { selection) }\end{array}$ & $\begin{array}{l}\text { Penetration } \\
\text { condition }\end{array}$ \\
\hline $\begin{array}{l}\text { Wang et al } \\
2011[74]\end{array}$ & NA & $\begin{array}{l}\text { Alluminium } \\
\text { Alloy }\end{array}$ & GTAW & $\begin{array}{l}\text { Wavelet - } \\
\text { Feature } \\
\text { extraction- } \\
\text { GA }\end{array}$ & ANN & $\begin{array}{l}\text { Depth Of } \\
\text { Penetration } \\
\text { Classification }\end{array}$ \\
\hline $\begin{array}{l}\text { Sumesh et. al } \\
2015 \text { [52] }\end{array}$ & Butt Weld & $\begin{array}{l}\text { Carbon } \\
\text { Steel }\end{array}$ & GMAW & N/A & $\begin{array}{l}\text { 1. Statistical } \\
\text { feature } \\
\text { 2. Decision tree } \\
\text { \& random } \\
\text { forest } \\
\text { algorithm }\end{array}$ & $\begin{array}{l}\text { 1. Burn through } \\
\text { 2. Lack of } \\
\text { fusion }\end{array}$ \\
\hline $\begin{array}{l}\text { Zhang et al } \\
2015 \text { [56] }\end{array}$ & N/A & $\begin{array}{l}\text { Alluminium } \\
\text { Alloy }\end{array}$ & GMAW & $\begin{array}{l}\text { Signal } \\
\text { Normalizati } \\
\text { on (Time } \\
\text { Domain } \\
\text { Signal) } \\
\text { Power } \\
\text { Spectrum } \\
\text { Density } \\
\text { (Freq } \\
\text { Domain) }\end{array}$ & $\begin{array}{ll}\text { Classification } \\
\text { 1. } & \text { RMS } \\
\text { 2. } & \text { Variance } \\
\text { 3. } & \text { Kurtosis }\end{array}$ & $\begin{array}{ll}\text { 1. } & \text { Porosity } \\
\text { 2. } & \text { Seam } \\
\text { penetration } \\
\text { 3. } & \text { local cavity }\end{array}$ \\
\hline
\end{tabular}


Table 2. Summary of studies associates with the use of the air-borne acoustic method for monitoring and assessing the weld quality during the arc welding process (cont.)

\begin{tabular}{|c|c|c|c|c|c|c|}
\hline Author & Methodology & Material & $\begin{array}{l}\text { Welding } \\
\text { Type }\end{array}$ & $\begin{array}{c}\text { Pre- } \\
\text { Processing }\end{array}$ & Analysis & $\begin{array}{c}\text { Type of Defect/ } \\
\text { phenomena }\end{array}$ \\
\hline $\begin{array}{l}\text { Lv et al } 2017 \\
{[78]}\end{array}$ & N/A & N/A & GTAW & $\begin{array}{l}\text { Auditory } \\
\text { Attention } \\
\text { (Region of } \\
\text { Interest) }\end{array}$ & 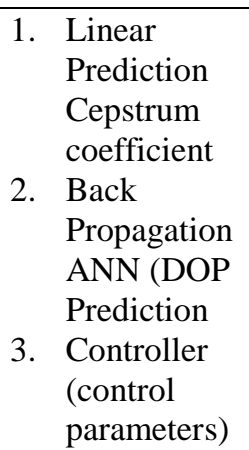 & $\begin{array}{l}\text { Depth of } \\
\text { Penetration }\end{array}$ \\
\hline $\begin{array}{l}\text { Yusof et al } \\
2017 \text { [68] }\end{array}$ & Butt weld & $\begin{array}{l}\text { API 5L X70 } \\
\text { steel }\end{array}$ & GMAW & HНT & Energy & $\begin{array}{l}\text { Porosity } \\
\text { Detection }\end{array}$ \\
\hline $\begin{array}{l}\text { Fidali } 2018 \\
\text { [86] }\end{array}$ & Butt Weld & $\begin{array}{l}\text { Steel } \\
\text { S235JR }\end{array}$ & GMAW & N/A & 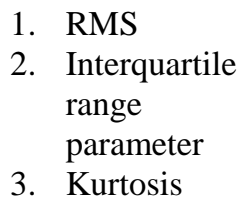 & Instabilities \\
\hline
\end{tabular}

\section{APPLICATION ACOUSTIC METHOD FOR MONITORING LASER WELDING PROCESS}

\section{Source of Acoustic Signals in Laser Welding Process}

In case of the laser welding process, the generations of structure-borne acoustic signals are commonly found from crack formation, porosity, phase transformation and the back-reflected laser [62, 63]. Like in the arc welding area of studies, up to now, the studies connected to the source of the structure-borne acoustic signal during both types of welding process are not involving mathematical model, except back-reflected laser which has been explained by Weerasinghe et al.,[92]. In their study, it is explained that at some point, when the laser beam hit the workpiece surface, some of the transmitted beam energy is absorbed or reflected. Back reflected laser would turn into amplified damped oscillation as it enters the laser cavity. The following equation could explain the frequency of this oscillation:

$$
f=\frac{1}{2 \pi}\left|\frac{\left(\frac{g}{g_{e}}-1\right)}{t_{s} t_{c}}\right|^{\frac{1}{2}} \quad \mathrm{~Hz}
$$

and the damping coefficient is given by:

$$
T_{d}=\frac{2 t_{s}}{\left(\frac{g}{g_{e}}\right)} S
$$

Where $g, g_{e}, t_{s}$ and $t_{c}$ could be described as the unsaturated gain, gain at the threshold, a lifetime of the upper laser level, and photon cavity lifetime respectively.

Due to the different process mechanism, phenomena such as plasma plume formation, weld pool and keyhole oscillation, as well as the gas jet pulsation that causing pressure variations in weld zone becomes the main sources of the air-borne acoustic signal during the laser welding process [93]. Previous researches show good agreement with this statement, whereas the acquired sound has proved to be significantly related to those phenomena. However, not many scholars try to present this correlation through a numerical analysis due to the fact that many sources influence acoustic generation. In conjunction, Asfar [94] emphasized that the question of how sound generated from laser welding process are still complex to answer. Still, many scholars agreed that it is due to violent plume fluctuation which causing surrounding air displaced rapidly and propagate as an acoustic wave. More detail explanation regarding this phenomenon has been explained through the mathematical model by Dowling et al [95]. In their work, it was explained that the evaporation process could be modelled by vapour creation rate, $m$, which involve parameter such as volume fraction occupied by newly created vapour, $\beta$, and vapour density $\rho_{m}$ as shown below

$$
m=\frac{\partial}{\partial t}\left(\beta \rho_{m}\right)
$$


When the formed vapour is assumed to displace the ambient air as it streams out from the keyhole, then the mass density in a control volume above the keyhole is given by:

$$
\rho=\beta \rho_{m}+(1-\beta) \rho_{f}
$$

whereas in this equation, the density of the ambient fluid is denoted as $\rho_{f}$. Under this circumstance, a differential equation could be written as:

$$
\frac{1}{c^{2}} \frac{\partial p^{\prime}}{\partial t}-\nabla^{2} p^{\prime}=\rho_{0} \frac{\partial_{2} \beta}{\partial t^{2}}
$$

Under the assumption that evaporant source is relatively small to the acoustic wavelength in ambient air, acoustic pressure at distance $r$ from the source could be written as the following equation:

$$
p^{\prime}(r, t)=\rho_{0} \frac{\partial^{2}}{\partial t^{2}}\left(\frac{\beta\left(t-\frac{r}{c}\right)}{4 \pi r}\right)
$$

in which $c$ is the speed of sound, and the mass flow rate from the keyhole is proportional to the time derivatives of the volume fraction $\beta$ :

$$
\frac{m}{\rho_{m}}=\frac{\partial}{\partial t}(\beta)
$$

The sound pressure perturbation from unsteady evaporation relates to the time derivative of the evaporation mass flowrate. As the radius distance from the plume increases further, the sound pressure decrease.

\section{Application of Acoustic Methods in Laser Welding Process Monitoring}

In studies involving monitoring of weld quality or condition during the laser welding process, the application of structure-borne acoustic method also have been reported. Past evidence shows that this method is capable of detecting defects such as melting, solidification [9], back-reflected laser [92, 96] as well as porosity [55]. However, the application of this method in both laser and arc welding process recorded lack in number. This might be due to the structure-borne nature of the emitted signal which needs the sensor to be directly contacted on the surface of the workpiece as shown in Figure 1, which makes it difficult when dealing with high-temperature process like welding.

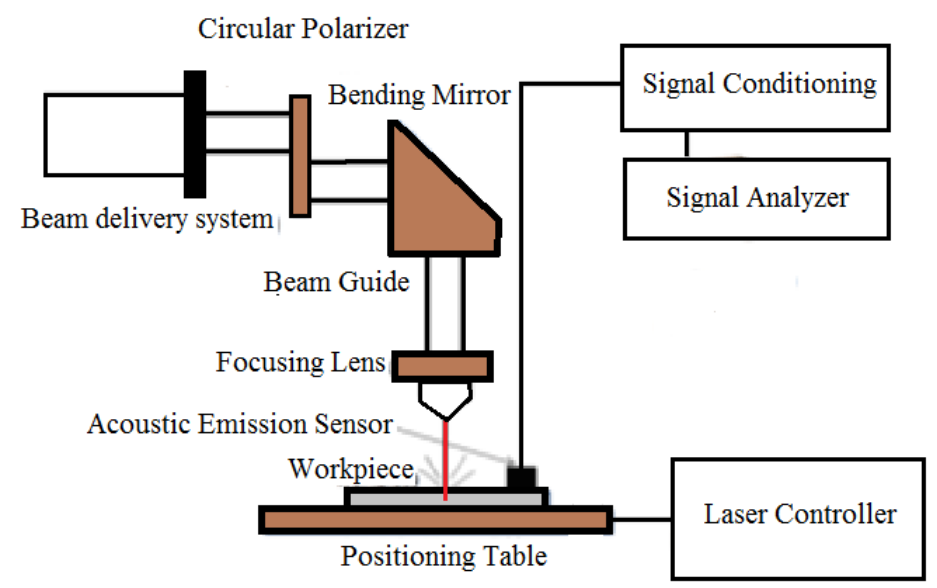

Figure 1. Acoustic emission system setup during laser welding process [9]

Disregard the use of structure-borne acoustic signal, the use of air-borne acoustic signal as a medium for monitoring the laser welding process also has been explored since the past several decades. Apparently, many studies agreed that the plasma plume formation and weld pool oscillation are two phenomena which are the significant sources of sound during the laser welding process. Therefore, any instability on those occasions would consequently affect the weld quality thence it is vital to monitor those phenomena. 
Table 3. Summary of studies associates with the use of the acoustic method for monitoring and assessing the weld quality during the laser welding process

\begin{tabular}{|c|c|c|c|c|c|c|}
\hline Author & Methodology & Material & Signal Type & $\begin{array}{c}\text { Pre- } \\
\text { Processing }\end{array}$ & Analysis & $\begin{array}{c}\text { Detected } \\
\text { phenomena }\end{array}$ \\
\hline $\begin{array}{l}\text { Weerasinghe et } \\
\text { al } 1990 \text { [92] }\end{array}$ & $\begin{array}{l}\text { 1. } \mathrm{CO}_{2} \text { Laser } \\
\text { 2. Bead on } \\
\text { plate }\end{array}$ & N/A & $\begin{array}{l}\text { Acoustic } \\
\text { emission }\end{array}$ & N/A & $\begin{array}{l}\text { 1. AE RMS } \\
\text { 2. AE frequency }\end{array}$ & Back reflected laser \\
\hline Li 2002 [96] & $\mathrm{CO}_{2}$ laser & Mild Steel & $\begin{array}{l}\text { 1. Acoustic } \\
\text { 2. Acoustic } \\
\text { Emission }\end{array}$ & N/A & $\begin{array}{l}\text { 1. AE RMS } \\
\text { 2. AE frequency }\end{array}$ & $\begin{array}{l}\text { 1. Back-reflected } \\
\text { laser } \\
\text { 2. Plasma behavior }\end{array}$ \\
\hline $\begin{array}{l}\text { Lee et al } 2014 \\
\text { [9] }\end{array}$ & $\begin{array}{l}\text { 1. NdYAG } \\
\text { laser } \\
\text { 2. Pulse mode }\end{array}$ & $\begin{array}{l}304 \text { stainless } \\
\text { steel }\end{array}$ & $\begin{array}{l}\text { Acoustic } \\
\text { emission }\end{array}$ & N/A & $\begin{array}{l}\text { 1. AE signal } \\
\text { values at } \\
\text { selected } \\
\text { frequency } \\
\text { band } \\
\text { 2. Back } \\
\text { Propagation } \\
\text { ANN }\end{array}$ & $\begin{array}{l}\text { 3. Melting } \\
\text { 4. Solidificaton } \\
\text { 5. JoinedCondition }\end{array}$ \\
\hline $\begin{array}{l}\text { Lewis \& Dixon } \\
1984 \text { [97] }\end{array}$ & $\begin{array}{l}\text { 1. NdYAG } \\
\text { laser } \\
\text { 2. Pulse mode }\end{array}$ & $\begin{array}{l}304 \text { stainless } \\
\text { steel }\end{array}$ & Acoustic & N/A & Velocity & $\begin{array}{l}\text { Plasma Shock } \\
\text { Wave } \\
\text { (categorization) }\end{array}$ \\
\hline $\begin{array}{l}\text { Conesa et al } \\
2004[98]\end{array}$ & $\begin{array}{l}\text { 1. NdYAG } \\
\text { laser } \\
\text { 2. Pulse mode } \\
\text { 3. Ablation }\end{array}$ & Alluminium & Acoustic & N/A & $\begin{array}{l}\text { Acoustic } \\
\text { EnergySpectrum }\end{array}$ & $\begin{array}{l}\text { 1. Irradiance trend } \\
\text { 2. Plasma Expansion }\end{array}$ \\
\hline $\begin{array}{l}\text { Farson et al } \\
1999 \text { [99] }\end{array}$ & $\begin{array}{l}\text { 1. } \mathrm{CO}_{2} \text { Laser } \\
\text { 2. Continuous } \\
\text { mode } \\
\text { 3. LapJoint }\end{array}$ & Carbon steel & Acoustic & N/A & $\begin{array}{l}\text { Root Mean } \\
\text { Square }\end{array}$ & $\begin{array}{l}\text { Depth of penetration } \\
\text { (classification) }\end{array}$ \\
\hline $\begin{array}{l}\text { Duley \& Mao } \\
1994 \text { [100] }\end{array}$ & $\begin{array}{l}\text { 1. } \mathrm{CO}_{2} \text { Laser } \\
\text { 2. Bead on } \\
\text { plate }\end{array}$ & $\begin{array}{l}\text { Alluminum } \\
1100\end{array}$ & Acoustic & $\begin{array}{l}\text { Bandpass } \\
\text { filter }\end{array}$ & $\begin{array}{l}\text { Amplitude of Fre } \\
\text { quency domain } \\
\text { signal }\end{array}$ & $\begin{array}{l}\text { 1. Depth of } \\
\text { penetration } \\
\text { 2. Surface condition }\end{array}$ \\
\hline $\begin{array}{l}\text { Farson et al } \\
1996[101]\end{array}$ & $\begin{array}{l}\text { 1. Continuous } \\
\text { mode } \\
\text { 2. Lap joint }\end{array}$ & $\begin{array}{l}304 \text { stainless } \\
\text { steel }\end{array}$ & Acoustic & $\begin{array}{l}\text { Short Time } \\
\text { Fourier } \\
\text { Transform }\end{array}$ & $\begin{array}{l}\text { 1. Band power } \\
\text { 2. Artificial } \\
\text { Neural } \\
\text { Network } \\
\text { (Classification } \\
\text { penetration } \\
\text { condition) } \\
\text { 3. Spectrum } \\
\text { energy (Gap } \\
\text { analysis) }\end{array}$ & $\begin{array}{l}\text { 1. Depth of } \\
\text { penetration } \\
\text { 2. Gap }\end{array}$ \\
\hline $\begin{array}{l}\text { Szymanski et al } \\
2001 \text { [102] }\end{array}$ & $\begin{array}{l}\text { 1. } \mathrm{CO}_{2} \text { laser } \\
\text { 2. Continuous } \\
\text { wave }\end{array}$ & $\begin{array}{l}\text { 1. Mild steel } \\
\text { 2. Stainless } \\
\text { steel }\end{array}$ & Acoustic & N/A & $\begin{array}{l}\text { Spectrum } \\
\text { analysis }\end{array}$ & $\begin{array}{l}\text { plasma plume } \\
\text { oscillation }\end{array}$ \\
\hline $\begin{array}{l}\text { Hoffman et al } \\
2002[11]\end{array}$ & N/A & $\begin{array}{l}\text { Austenitic } \\
\text { steel }\end{array}$ & Acoustic & N/A & $\begin{array}{l}\text { Mathematical } \\
\text { Model (Verified } \\
\text { by experiment) }\end{array}$ & $\begin{array}{l}\text { plasma plume } \\
\text { oscillation }\end{array}$ \\
\hline $\begin{array}{l}\text { Huang \& } \\
\text { Kovacevic } \\
2009[62]\end{array}$ & $\begin{array}{l}\text { 1. Continuous } \\
\text { mode } \\
\text { 2. Lap joint }\end{array}$ & $\begin{array}{l}\text { DP980 HS } \\
\text { steel }\end{array}$ & Acoustic & $\begin{array}{l}\text { Spectral } \\
\text { Subtraction }\end{array}$ & $\begin{array}{l}\text { 1. Band power } \\
\text { 2. Sound } \\
\text { Pressure } \\
\text { Deviation }\end{array}$ & $\begin{array}{l}\text { Depth of penetration } \\
\text { (classification) }\end{array}$ \\
\hline $\begin{array}{l}\text { Huang \& } \\
\text { Kovacevic } \\
2011[63]\end{array}$ & $\begin{array}{l}\text { 1. Continuous } \\
\text { mode } \\
\text { 2. Lap joint }\end{array}$ & $\begin{array}{l}\text { DP980 HS } \\
\text { steel }\end{array}$ & Acoustic & $\begin{array}{l}\text { Spectral } \\
\text { Subtraction }\end{array}$ & $\begin{array}{l}\text { 1. Band Power } \\
\text { 2. Sound } \\
\text { Pressure } \\
\text { Deviation } \\
\text { 3. Artificial } \\
\text { Neural } \\
\text { Network } \\
\text { 4. Multiple } \\
\text { regression }\end{array}$ & $\begin{array}{l}\text { Depth of penetration } \\
\text { (estimation model) }\end{array}$ \\
\hline
\end{tabular}


Table 3. Summary of studies associates with the use of the acoustic method for monitoring and assessing the weld quality during the laser welding process (cont.)

\begin{tabular}{|c|c|c|c|c|c|c|}
\hline Author & Methodology & Material & Signal Type & $\begin{array}{c}\text { Pre- } \\
\text { Processing }\end{array}$ & Analysis & $\begin{array}{c}\text { Detected } \\
\text { phenomena }\end{array}$ \\
\hline $\begin{array}{l}\text { Khosroshahi et } \\
\text { al } 2010 \text { [61] }\end{array}$ & $\begin{array}{l}\text { 1. } \mathrm{CO}_{2} \text { laser } \\
\text { 2. Pulse mode } \\
\text { 3. Ablation }\end{array}$ & $\begin{array}{l}316 \mathrm{~L} \\
\text { Stainless } \\
\text { Steel }\end{array}$ & Acoustic & N/A & $\begin{array}{l}\text { Amplitude of } \\
\text { pulse sound } \\
\text { signal }\end{array}$ & $\begin{array}{l}\text { 1. Mass removal } \\
\text { (ablation) } \\
\text { 2. Plasma plume } \\
\text { formation }\end{array}$ \\
\hline $\begin{array}{l}\text { Sansan Ao et al } \\
2015 \text { [59] }\end{array}$ & NdYAG laser & Iron & Acoustic & N/A & $\begin{array}{l}\text { Mathematical } \\
\text { Model (Verified } \\
\text { by experiment) }\end{array}$ & $\begin{array}{l}\text { Weld Pool } \\
\text { Oscillation frequency }\end{array}$ \\
\hline $\begin{array}{l}\text { Zhen Luo et al } \\
2016 \text { [49] }\end{array}$ & $\begin{array}{l}\text { 1. NdYAG } \\
\text { laser } \\
\text { 2. Continuous } \\
\text { mode }\end{array}$ & Steel plate & Acoustic & $\begin{array}{l}\text { N/A (use } \\
\text { sound } \\
\text { proofing } \\
\text { equipment to } \\
\text { avoid noise) }\end{array}$ & $\begin{array}{l}\text { 1. Acoustic } \\
\text { pressure level } \\
\text { 2. Time delay } \\
\text { recognition }\end{array}$ & Burn through \\
\hline $\begin{array}{l}\text { Chang Je Lee } \\
2015 \text { [53] }\end{array}$ & $\begin{array}{l}\text { 1. } \mathrm{CO}_{2} \text { Laser } \\
\text { 2. Continuous } \\
\text { mode } \\
\text { 3. LapJoint }\end{array}$ & $\begin{array}{l}\text { AH } 36 \\
\text { structural } \\
\text { steel }\end{array}$ & $\begin{array}{l}\text { 1. Acoustic } \\
\text { 2. Photodiode }\end{array}$ & $\begin{array}{l}\text { Bandpass } \\
\text { filter }\end{array}$ & $\begin{array}{l}\text { Root Mean } \\
\text { Square }\end{array}$ & $\begin{array}{l}\text { 1. Spatter } \\
\text { 2. Humping }\end{array}$ \\
\hline $\begin{array}{l}\text { Farson et al } \\
1998 \text { [103] }\end{array}$ & $\begin{array}{l}\text { 1. } \mathrm{CO}_{2} \text { Laser } \\
\text { 2. Continuous } \\
\text { mode } \\
\text { 3. ButtJoint }\end{array}$ & Carbon steel & $\begin{array}{l}\text { 1. Acoustic } \\
\text { 2. Optical } \\
\text { 3. Plasma }\end{array}$ & N/A & $\begin{array}{l}\text { Linear } \\
\text { Discriminant } \\
\text { analysis }\end{array}$ & $\begin{array}{l}\text { Depth of penetration } \\
\text { (Cassification) }\end{array}$ \\
\hline $\begin{array}{l}\text { Gu \& Duley } \\
1996[54]\end{array}$ & $\begin{array}{l}\text { 1. } \mathrm{CO}_{2} \text { Laser } \\
\text { 2. Lap } \\
\text { joint,Butt } \\
\text { joint, Bead } \\
\text { on plate }\end{array}$ & $\begin{array}{l}\text { Mild \& } \\
\text { Galvanized } \\
\text { steel }\end{array}$ & Acoustic & N/A & $\begin{array}{l}\text { 1. Sum of a } \\
\text { squared } \\
\text { standard } \\
\text { deviation } \\
\text { 2. Discriminant } \\
\text { function }\end{array}$ & $\begin{array}{l}\text { 1. Depth of } \\
\text { penetration } \\
\text { 2. Heat affected } \\
\text { zone } \\
\text { 3. Porosity }\end{array}$ \\
\hline $\begin{array}{l}\text { Farson \& Kim } \\
\text { (1999) [60] }\end{array}$ & $\begin{array}{l}\text { 1. } \mathrm{CO}_{2} \text { Laser } \\
\text { 2. Bead on } \\
\text { plate }\end{array}$ & $\begin{array}{l}\text { Low carbon } \\
\text { steel }\end{array}$ & $\begin{array}{l}\text { 1. Acoustic } \\
\text { 2. Optical }\end{array}$ & N/A & Prediction model & $\begin{array}{l}\text { Acoustic and plasma } \\
\text { emission trend based } \\
\text { on material } \\
\text { evaporation and } \\
\text { formation of ionized } \\
\text { plume }\end{array}$ \\
\hline $\begin{array}{l}\text { Ao et al } 2010 \\
{[48]}\end{array}$ & $\begin{array}{l}\text { 1. NdYAG } \\
\text { laser } \\
\text { 2. Bead on } \\
\text { plate }\end{array}$ & $\begin{array}{l}\text { Cold rolled } \\
\text { steel }\end{array}$ & Acoustic & N/A & $\begin{array}{l}\text { 1. Frequency } \\
\text { Domain } \\
\text { 2. PCA-ICA }\end{array}$ & Blowholes \\
\hline $\begin{array}{l}\text { Yusof et al } \\
\text { (2017) [67] }\end{array}$ & $\begin{array}{l}\text { 1. Fibre Laser } \\
\text { 2. Pulse mode } \\
\text { 3. Bead on } \\
\text { Plate }\end{array}$ & $\begin{array}{l}\text { Boron Steel } \\
22 \mathrm{MnB} 5\end{array}$ & Acoustic & & $\begin{array}{l}\text { Sound Pressure } \\
\text { Level }\end{array}$ & Depth of penetration \\
\hline
\end{tabular}

Table 3 shows the summary of earlier studies related to the use of acoustic method for monitoring laser welding process. In early work by Lewis and Dixon [97], an attempt to dig deeper comprehend on the emitted plasma behaviour from laser-material interaction has been made with an aided from the acquired sound data. Specifically, in their experiment, the plasma shock wave velocity produced from the pulse-mode laser welding process was obtained from the acquired acoustic signals. By calculating the plasma shock wave velocity, it could be characterized whether it was laser supported combustion (LSC) or laser supported detonation (LSD) type. This could give some clue about the laser energy absorption status during the coupling process. Extended to this work, Conesa et al., [98] reported that the transition from LSD regime to laser supported radiation (LSR) regime was noticeable by remarkable trend of the acoustic signal energy for irradiance value. This was illustrated in Figure 2. A similar trend has also been presented by Khosroshahi et al., [61] but in addition to their work, the relation between acoustic signal amplitude was analytically modelled. In another effort, Farson and Kim [60] have formulated the analytical model for the generation of the air-borne acoustic signal. According to their formulation, the generated sound pressure was influenced by the vapour flow rate since the surrounding air was displaced by the vapour emanating from the keyhole. Szymanski et al., [102] and Hoffman et al., [11] also have confirmed this statement in both of their mathematical and experimental analysis. Moreover, in their analytical work, the zone occupied by plasma was considered as an impenetrable pulsating sphere. Regardless of plasma plume formation, the acoustic signal could also emerge from the weld pool or keyhole oscillation. Due to this fact, Ao et al. [59], make an effort to predict the oscillatory frequency from the modelling process of weld with different penetration depth induced by different weld speed. The result from their work shows that from two-dimensional modelling process, the simulated weld pool oscillation frequency deviates around $4 \%$ from the experimental results. 


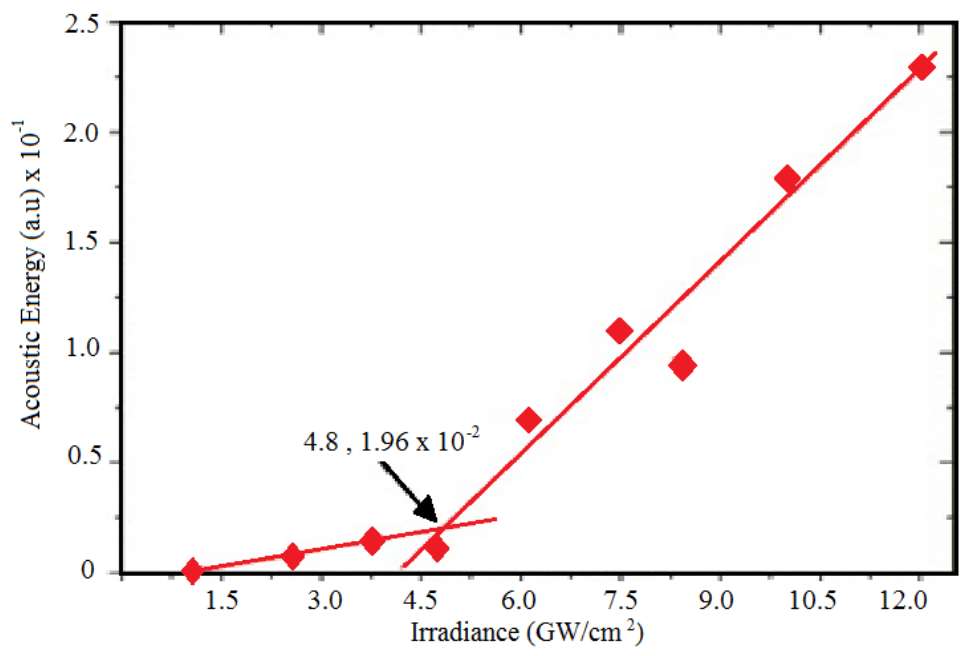

Figure 2. Acoustic energy response from the increment of irradiant value [98]

In the earlier statement, it was emphasized that any instability of physical phenomena such as plasma plume formation and weld pool or keyhole oscillation that emerges from the laser welding process could lead to the formation of the defect. Hence, despite implying an airborne acoustic signal to gain a deeper understanding of those physical phenomena, the relation between the behaviours of the acquired acoustic signal with weld condition also has been investigated. Several evident proved that information embedded in the received acoustic signal could be significantly used to determine the weld condition as well as detecting and locating the defect. For instance, the correlation between acoustic spectral features and depth of penetration has been investigated by Duly and Mao [100]. In their study, a test was done on $2 \mathrm{~mm}$ thickness Aluminium 1100 using $\mathrm{CO}_{2}$ laser welding while the captured sound signal was low passed filtered with the cut-off frequency of $10 \mathrm{kHz}$. According to the result, the emission of the acoustic signal was dominant between $0-1 \mathrm{kHz}$ at low incident laser intensity. In contrast, peak within $9 \mathrm{kHz}$ to $10 \mathrm{kHz}$ was found increasing in its amplitude simultaneously with gaining laser intensity. Consequently, the overall amplitude arises with extended depth of penetration. Furthermore, the frequency component between $3-9 \mathrm{kHz}$ was perceived to be associated with closure of the keyhole over part of its length due to hydrodynamical instabilities. Thence, the partial closure of keyhole could be detected by observing the spectrum within this frequency range. Similar work also has been reported by Farson et al., [101] whereas the characteristic of the acoustic signal was analyzed to identify the weld quality which qualitatively determined by the depth of penetration and gapping. The experiment was done on 304 stainless steel plate. Unlike the reported previous works, the time-frequency approach was taken by implying Short Time Fourier Transform to identify the significant frequency range with respect to the weld quality. Through the analysis, the significant range was recorded to be within $1 \mathrm{kHz}$ to 2 $\mathrm{kHz}$ in which its energy dropped when insufficient penetration detected. This trend could be referred to in Figure 3. Meanwhile, by calculating the total signal strength, a clear distinction between the gapped weld and normal weld could be observed. Not to be outdone, the use of an Artificial Neural Network to classify the penetration status also has been demonstrated. With the help of ANN, full penetration and partial penetration weld could be distinguished in a different group.

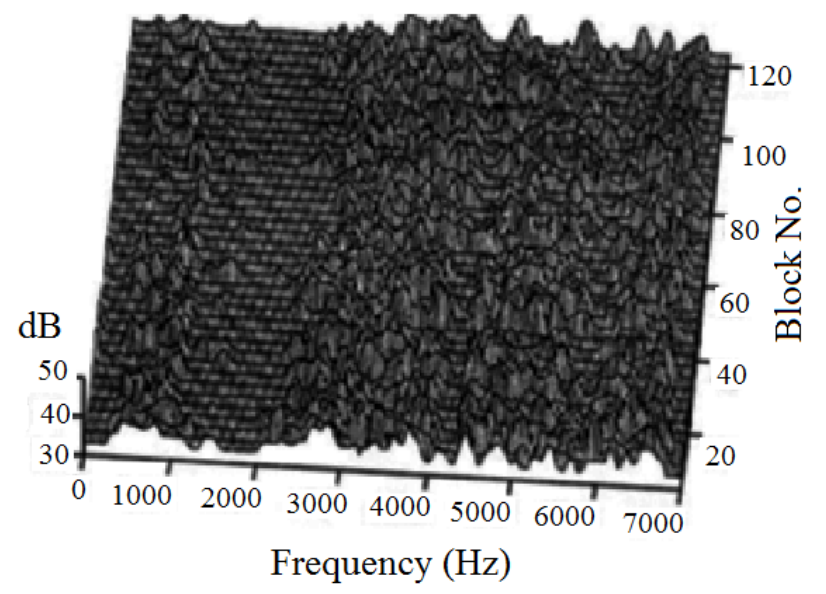

Figure 3. Spectrogram of the acquired sound signal [101]

As reported in earlier work, it could be drawn into a conclusion that monitoring the penetration status by analyzing the acquired sound is possible. However, the reported work emphasizes on the cases involving a narrow range of welding parameters which make it challenging to ensure the robustness of the observation study. Hence, in the extended study by 
Farson et al [103], the investigation was done in a broader range of power and travel speed. As a result, the classification of penetration status could be extended into moderately full penetration as an addition to the present full and partial penetration class. In this work, it was described that the Root Mean Square (RMS) of the acoustic signal was high at moderate-full penetration, and it eventually decreasing as the penetration status felt into partial penetration class. Disparate from the other works, in another work by Farson and Kim, [60], more attention has been paid in identifying the penetration status during high power or keyhole welding. Supported by measured optical charged particle data, the depth of penetration status could be classified into full penetration, overheat penetration and half penetration. In this study, the overheat penetration referred to weld produced by excessive linear heat input which consequently caused a significant level of top surface concavity and more massive heat-affected zone. Meanwhile, the classification was done through Linear Discriminant Analysis. Similar findings also have been described by Gu and Duley [54] whereas, through discriminant analysis, three groups of penetration status was successfully classified. Moreover, in their work, it was found that by determining the sum of a squared standard deviation, bad quality weld could be easily identified. In another work by Huang and Kovacevic [62], the classification was done through the analysis of both time and frequency domain signal characteristic. Uniquely, in their research, the spectral subtraction method was demonstrated to diminish the influence of noise in the analysis result, and the outcome was found to be promising. As an observation made onto the captured time domain, it was reported that the overall sound pressure increase aligns with the growth in penetration depth. Meantime, the power density of the frequency spectrum from $500 \mathrm{~Hz}$ to $1500 \mathrm{~Hz}$ was large for full penetration case as compared to half penetration. Unlike other work which emphasizes on continuous mode laser welding, Yusof et al. [67] have paid more attention to how sound characteristic from pulse mode laser welding could be correlated with the weld penetration. According to their work, it was revealed that the transient behaviour of sound signal was emerging from the process and sound pressure level shows non-linear behaviour for the change in weld depth when the pulse energy reaches a certain value

Sensibly, classifying the penetration status is insufficient for the case where the depth of penetration needs to be quantitatively measured. Thence, to overcome the drawback, in their extended work, Huang \& Kovacevic [63] have utilized the Artificial Neural Network analysis in an attempt to quantitatively characterize the relation between the captured sound and the depth of penetration. In this extended work, the multiple regressions also have been applied as the comparison with ANN result. According to the obtained results, by giving the value of sound pressure deviation and band power as an input to the model, the best neural network model was found to be able to predict the depth of penetration with standard deviation error of $8.91 \%$. Meanwhile, for the multiple regression model, the standard deviation error was recorded to be $8.25 \%$.

Despite classification and characterization of the weld penetration depth, studies were also extended into the detection of other types of defect. For instance, Zhen Luo et al., [49] exhibit the use of the multiple numbers of microphones to detect and locate the burn through phenomena. In their study, the soundproof equipment was specially design and used to avoid the influence of noise during the sound acquisition process. Based on the illustrated result, the use of soundproof equipment proved to influence the outcome significantly. It was noted that the detection and location of burn trough defect based on the sound pressure level and time delay recognition analysis was done with a lesser error when soundproof equipment was used. In another work, the use of the acoustic method also reported being useful to detect other types of defect such as underfill and humping [53]. In this work, the effect of $\mathrm{Zn}$ coating thickness, and the gap between the lap joint, to the degree of those defects were monitored based on the acquired sound signal. From the drawn result as shown in Figure 4, the amount of spatter which influences the existence of both underfil and humping was consistent between 0.08 to $0.2 \mathrm{~mm}$ gap depending on the coating thickness. Simultaneously, within the same range, the recorded RMS of the sound signal was significantly changed. Moreover, the analysis of frequency spectrums shows that the dominant frequency lies within $1 \mathrm{kHz}$ for both 15 micrometres and 30 micrometres $\mathrm{Zn}$ coating thickness. However, the amplitude of spectrum appeared in a descending pattern when coating thickness increased. This is because the immense thickness value contributes to high vapour pressure which consequently suppressed periodic motion of keyhole. In addition, the amplitude of the time-domain sound signal, which filtered at the dominant frequency also shows deviating values from $22 \mathrm{~V}$ to $38 \mathrm{~V}$ when the defect exists. In another unique study, Ao et al., [48] imply the blind source separation technique which combining principal component analysis (PCA) and independent component analysis (ICA). Through blind source separation analysis, the acoustic signal successfully decomposed into cooling and keyhole component, and the existence of blowholes was detected. 
Lap welding: $f \mathrm{~d}=-2 \mathrm{~mm}, \mathrm{P}=6 \mathrm{~kW}, \mathrm{v}=1 \mathrm{~m} / \mathrm{mm}, \mathrm{Gc}=0.2 \mathrm{~mm}, \mathrm{He}$ shield $(\mathrm{Q}=25 \mathrm{~L} / \mathrm{min})$

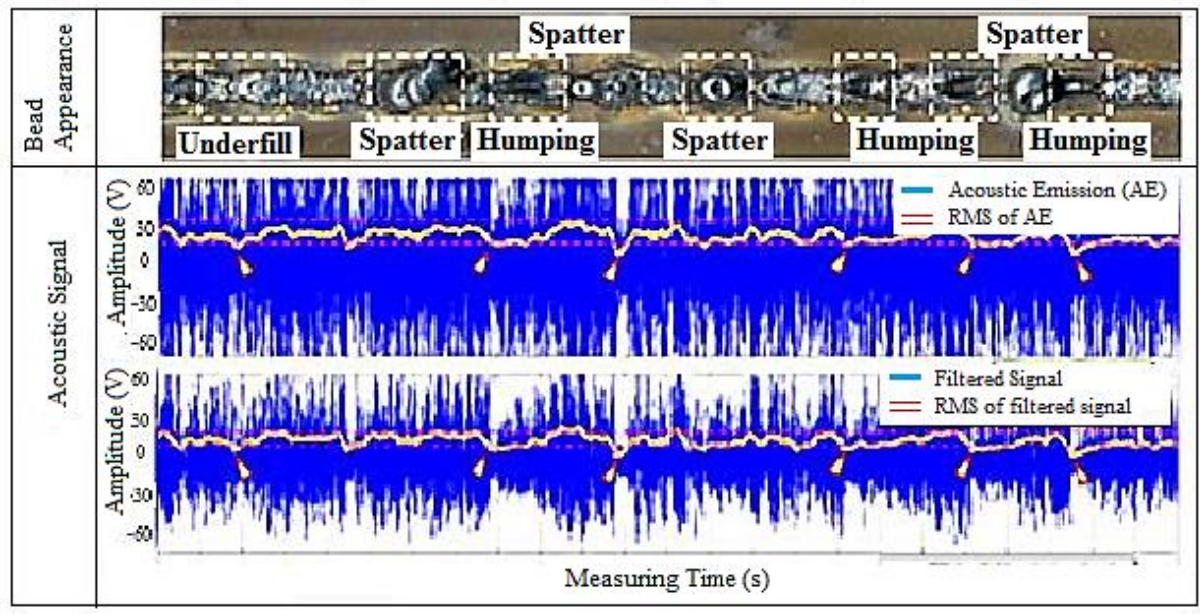

Figure 4. RMS of the acoustic signal during the presence of damage [53]

\section{FUTURE RESEARCH ON THE ADVANCEMENT OF ACOUSTIC METHOD FOR WELDING MONITORING}

Based on the reviewed studies, it is learned that there were numerous studies associate with the use of acoustic signal for monitoring welding process, which covers a wide variety of research scopes. By general comparison, it could be observed that the use of the structure-borne acoustic method in both arc and laser welding cases recorded least in number as compared to the air-borne method. As previously outlined, it might be due to the difficulties in acquiring the data from structure-borne acoustic wave under high temperature during the process. If all the scopes and aims of all studies involving both arc and laser welding are combined, it could be summarized, as shown in Figure 5.

As abridged in the chart illustrated in Figure 5, studies could be classified into experimental- and simulation-based study. In the experimental-based research, it covers a wide variety of welding type for both arc and laser welding case. Meanwhile, in analyzing the acquired sound signal, some of the work emphasizes the significance of pre-processing method in reducing the influence of noise captured during acoustic signal acquisition process before extracting the signal features. Uniquely, the overviews show that this approach only involves the study of the air-borne acoustic signal. This is because an air-borne type of signal is easily interrupted by the ambient noise [64] while the high-frequency nature of structure-borne makes it difficult to be influenced by noise. On another way round, some of the researches tend to directly find the ideal signal features without emphasizing on the pre-processing of the acquired signal. However, there was an agreement between both scopes of study in which both studies aimed to detect, locate, or classify the defect types as well as predict its trend. Distinctively, there was a study which focused on the adaptive control of the welding process based on the feedback from the acquired sound [78].

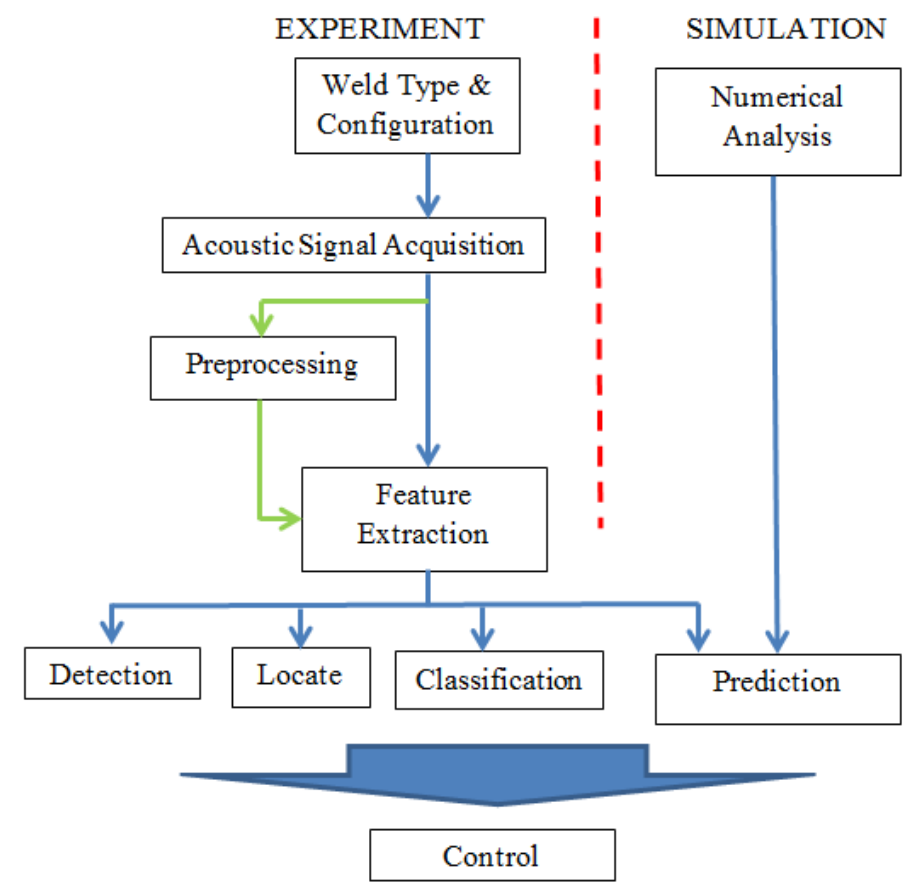

Figure 5. Summary of scopes of studies associates with the use of the acoustic method for monitoring the welding process 


\section{Type of Welding Process and Material}

As highlighted in the beginning part of this review, welding technology has recorded rapid growth since past few decades due to the increasing demand on this process. Overall look into the scope of studies, it could be observed that the studies involved various types of both arc and laser welding. In studies related with the arc weld, many studies focused on sound generation on common welding process like Gas Metal Arc Welding (GMAW) [50, 51, 58, 67, 72, 84, 86-88], Gas Tungsten Arc Welding (GTAW) [56, 78, 91] as well as Shielded Metal Arc Welding (SMAW) [52]. In some case, the use of a recently developed method such as Pulse-GMAW [51, 84, 88] and Variable Polarity Plasma Arc Welding (VPPAW) [90] also have been demonstrated. Wang et al [74] in their study have claimed that there is no significant difference in the behaviour of sound emerged from gas shielded welding process including GTAW and GMAW. The reason might be due to a nearly identical physical process involved in that type of welding process. However, as explained in the previous topic, some scholars $[69,72,79]$ agree that the sound generation in arc weld was strongly influenced by metal transfer or deposition as well as the molten pool behaviour. Since past decades, there were studies that have been made to comprehend the sound generation from both phenomena $[51,57,58,70]$. However, it was recorded lack in numbers. It is essential to get a deeper understanding on the sound generation from the behaviour of both metal deposition and molten pool since defect formation is originated from the instability of these phenomena thence more study in this area is needed.

Turning into the case of studies involving laser welding process, it could be observed that the studies were actually a sum of variation from different laser wave mode, laser type, workpiece material and also the joining configuration. From a more in-depth remark, it was perceived that lack of attempt is made to understand the emitted sound behaviour during pulse mode laser welding and how it could be correlated with the weld condition. A great deal of previous research into this area has focused on the trend of acoustic amplitude [61], energy [98] and also shock wave velocity [97] during the laser-material interaction. In these lines of works, particular attention has been made only to the emission of plasma plume induced by laser-material interaction. However, it is more significant if the other important phenomena such as weld pool or keyhole oscillation could be extracted from the acquired acoustic signal owing to the fact that the inter-correlation between those phenomena could lead to the formation of various defects. Due to the reason that the pulse-mode seam laser welding could possibly emit a cyclo-stationary random acoustic signal, extracting the information for the weld pool oscillation and plasma plume formation independently might be challenging. Thence, the feasible of decomposition method to be imposed to overcome the challenge need to be studied.

Apart from the welding type, there are also insufficient numbers of studies related to the sound generated during the welding process of the non-ferrous metal. Many studies in the past have done it on the Aluminum alloy [56, 74, 89-91, $98,100]$, but there are various types of non-ferrous metal available in industrial application. Theoretically, it is known that reaching the stability process for non-ferrous metal welding is quite challenging, and that is the main reason why monitoring approach is essential in this case. Under this fact, a depth understanding of the sound emission from the welding process of non-ferrous metal is necessary. Moreover, since the chemical compositions in the material more or less could influence the physical process such as solidification, phase transformation, plume density and keyhole oscillation, it is estimated that the behaviour of the emitted sound is also different and more challenging to be analyzed.

\section{Feature Extraction and Noise Elimination}

Basically, when the acquisition system captures the sound, the sampling process took place whereas the amplitude of sound at a specific instance was recorded, and the process was paused for a time interval called sampling period. This sampling process was repeated or cycled until the end of the acquisition process. According to the signal processing principal, the sampling period is determined by the sampling rate. Specifically, in the case of sound acquisition in the welding process, the sampling rate is defined as how many sound amplitude data point was sampled within 1 second. Accumulating all the reviewed work in the previous topic, it could be summarized that the sampling rate was set within 20k Sample/s to 50k Sample/s for the case of air-borne acoustic signal. Meanwhile, in studies related to structure-borne signal, the sampling rate was set up to 5M Sample/s. For example, if the sampling rate was set to be 20k Sample/s and welding process taken within 10 seconds to complete the process, the total sound amplitude data point in time domain signal is 200 000. In many cases involving the analysis of the time-domain signal, the sound amplitude characteristic was represented by its distribution trend. Statistical features will be extracted from this distribution in order to characterize the sound from different weld condition or process mechanism.

In earlier study, attempt in detecting, classifying or characterizing the defect from the acquired acoustic signal was made by obtaining the common statistical such as root mean squares [51, 53, 56, 86, 92, 96, 103], variance [56, 70], kurtosis [50, 51, 56, 58, 86, 88], and interquartile range [86]. Defects such as burn through [49, 50, 52, 59], cavity [56], lack of fusion [52] humping [53] and penetration condition [54,103] could be detect or characterize from the trend of those features. Apart from common statistical features, other features such as sound pressure level [49, 72, 87], sound pressure deviation $[62,63]$ and time of delay $[49,83]$ also have been demonstrated. Unlike the analysis of air-borne acoustic signal, the structure-borne acoustic emission is characterized by its specific features such as AE count, AE RMS, AE Energy and so forth $[9,82,84,92,96]$.

Despite the analysis of the time-domain signal, some authors have demonstrated the use of frequency spectrum features such as dominant frequency $[9,58,92,96,100]$ as well as the band power $[62,63,82,98,103]$ in solving their problem. This feature was found to be more significant as it does not influence by the noise from other frequency due to 
the reason that both feature only focused on the specific frequency span. However, in case some cases, the information of acoustic frequency at a specific time is needed. As the frequency domain analysis does not provide information about time, some studies use time-frequency analysis in their research. In some previous works, a method such as Short Time Fourier Transform [70], Wavelet analysis [74, 85, 91] and Hilbert Huang Transform [68] have been applied onto the acquired acoustic signal, and it was found to be significant in detecting defect in welding process.

Apart from implying time-frequency approach to extract the feature from it, this method could also be a tool for noise elimination as it is basically formed from the decomposition of the time domain signal into several component with different frequencies. By this reason, this method could possibly be a tool for noise elimination. According to You et al. [64], using acoustic method in monitoring welding process is challenging due to reason that acoustic signal is influenced by the harsh noise surrounding. Owing to this point, some scholars took effort to eliminate noise that occurs during the welding process by implement spectral subtraction $[62,63]$. However, it is vital to explore the capability of timefrequency approach in reducing the influence of noise during the process. Moreover, the time-frequency methods have shown a significant evolution since past several decades as many approaches have been introduced. Yet, it capability in solving noise problem as well as increasing the sensitivity of the acoustic method in detecting, characterizing, and locating the defect during welding process need to be studied.

\section{Defect Identification}

According to the summary in Figure 5, it was noticeable that studies which encompassing defect classification mainly involve the classification of penetration condition through the analysis of acquired sound [54, 99-101, 103]. In another group of literatures, detection of defect such as burn through [48, 49], porosity [54, 55], humping and spatter [53] was done by evaluating the feature behavior of the acquired sound. Moreover, the characterization of penetration size [63], plasma plume [60, 61], weld pool oscillation [59] as well as heat affected zone width [54] was also recorded. These studies proved that the acoustic method was capable to detect the presence of defect by online basis. However, in some cases, the detected defect can be ignored due to its small effect or severity. Hence, the identification of defect types and size is important in this case. In order to achieved this objective, deeper studies need to be done in order to understand how the predictive model could be developed to estimate the type and size of defect. For instance, the variation of surface roughness on the weld bead due to the existent of large underfil was proven to be the source of fatigue failure [104-106]. By developing predictive model, the size of underfill could be estimated and the process could be controlled by the online basis.

Overall, it could be concluded that the results were associates with defects classification, detection and also characterization. Pointing into another direction, it would be more significant if the type of defect could be identified from the analysis of sound signal. This is important because in quality control process, some types of defect are severe and the product needs to be rejected whilst some of it is allowable. To look after this direction, it is believed that the appropriate signal decomposition method needs to be implied. This is due to the reason that the source of specific defect might be clearly obtained from the decomposed signal.

\section{CONCLUSIONS}

To conclude, comprehensive reviews on the application of acoustic method in monitoring and assessing the weld quality have been given. The reviews cover the application of both structure- and air-borne acoustic on the liquid state welding process, and the scope of discussion converged on the arc and laser welding processes. Besides the reviews, explanation on how both type of acoustic signal could emerge from the process has been pointed out. In the last part of the review, the potential future advancement of this method is pointed out before the overall conclusion is made.

In essence, it is known that the development of monitoring system is important owing to its advantage in offering greater chance for process control in future. Previous researches proved that this method could significantly give the information regarding the weld condition by online basis. However, the recorded number of researches associate with this subject was still insufficient to deeply understand the emitted sound behavior during the laser welding process. This is important to ensure that this method could be used in wide variety of process parameter range, machine types, workpiece material as well as the condition of the environment itself. From the review, it could be concluded that there were still a lot of space for improvement needed for future work in order to improvise the robustness of this method.

\section{ACKNOWLEDGMENTS}

The authors would like to thanks to Universiti Malaysia Pahang for providing financial support under project no. RDU180314 for this particular work.

\section{REFERENCES}

[1] R. W. Messler Jr, Principles of welding: processes, physics, chemistry, and metallurgy: John Wiley \& Sons, 2008.

[2] M. Vural, "Welding Processes and Technologies," Comprehensive Materials Processing, vol. 6, pp. 3-48, 2014. 
[3] P. K. Giridharan and N. Murugan, "Optimization of pulsed GTA welding process parameters for the welding of AISI 304L stainless steel sheets," The International Journal of Advanced Manufacturing Technology, vol. 40, pp. 478-489, 2009.

[4] K. Y. Benyounis, A. G. Olabi, and M. S. J. Hashmi, "Multi-response optimization of CO2 laser-welding process of austenitic stainless steel," Optics \& Laser Technology, vol. 40, pp. 76-87, 2008.

[5] D. Kim, S. Rhee, and H. Park, "Modelling and optimization of a GMA welding process by genetic algorithm and response surface methodology," International Journal of Production Research, vol. 40, pp. 1699-1711, 2002.

[6] A. Kumar and S. Sundarrajan, "Optimization of pulsed TIG welding process parameters on mechanical properties of AA 5456 Aluminum alloy weldments," Materials \& Design, vol. 30, pp. 1288-1297, 2009.

[7] S. C. Juang and Y. S. Tarng, "Process parameter selection for optimizing the weld pool geometry in the tungsten inert gas welding of stainless steel," Journal of Materials Processing Technology, vol. 122, pp. 33-37, 2002.

[8] Y. S. Tarng, S. C. Juang, and C. H. Chang, "The use of grey-based Taguchi methods to determine submerged arc welding process parameters in hardfacing," Journal of Materials Processing Technology, vol. 128, pp. 1-6, 2002.

[9] S. Lee, S. Ahn, and C. Park, "Analysis of acoustic emission signals during laser spot welding of SS304 stainless steel," Journal of Materials Engineering and Performance, vol. 23, pp. 700-707, 2014.

[10] T. Purtonen, A. Kalliosaari, and A. Salminen, "Monitoring and adaptive control of laser processes," Physics Procedia, vol. 56, pp. 1218-1231, 2014.

[11] J. Hoffman, Z. Szymanski, J. Jakubowski, and A. Kolasa, "Analysis of acoustic and optical signals used as a basis for controlling laser - welding processes," Welding International, vol. 16, pp. 18-25, 2002.

[12] T. Le-Quang, S. A. Shevchik, B. Meylan, F. Vakili-Farahani, M. P. Olbinado, A. Rack, et al., "Why is in situ quality control of laser keyhole welding a real challenge?," Procedia CIRP, vol. 74, pp. 649-653, 2018.

[13] J. Shao and Y. Yan, "Review of techniques for on-line monitoring and inspection of laser welding," Journal of Physics: Conference Series, vol. 15, p. 101, 2005.

[14] D. Dickinson, J. Franklin, and A. Stanya, "Characterization of spot welding behavior by dynamic electrical parameter monitoring," Welding Journal, vol. 59, p. 170, 1980.

[15] C. S. Wu, T. Polte, and D. Rehfeldt, "Gas metal arc welding process monitoring and quality evaluation using neural networks," Science and Technology of Welding and Joining, vol. 5, pp. 324-328, 2000.

[16] V. Kumar, S. K. Albert, and N. Chandrasekhar, "Signal processing approach on weld data for evaluation of arc welding electrodes using probability density distributions," Measurement, vol. 133, pp. 23-32, 2019.

[17] H. Yong, W. Kehong, Z. Zhilan, Z. Xiaoxiao, and F. Jimi, "Stability evaluation of short-circuiting gas metal arc welding based on ensemble empirical mode decomposition," Measurement Science and Technology, vol. 28, p. 035006, 2017.

[18] Y. Huang, K. Wang, Q. Zhou, J. Fang, and Z. Zhou, "Feature extraction for gas metal arc welding based on EMD and timefrequency entropy," The International Journal of Advanced Manufacturing Technology, vol. 92, pp. 1439-1448, 2017.

[19] L. Li, D. J. Brookfield, and W. M. Steen, "Plasma charge sensor for in-process, non-contact monitoring of the laser welding process," Measurement Science and Technology, vol. 7, p. 615, 1996.

[20] B. Chao, Y. Lijun, X. Wenhao, and W. Langping, "An electrical detection of the fluctuating plasma of laser welding with a passive probe," Journal of Physics D: Applied Physics, vol. 45, p. 385202, 2012.

[21] S. Zhao, L. Yang, T. Liu, R. Yang, and J. Pan, "Analysis of plasma oscillations by electrical detection in Nd:YAG laser welding," Journal of Materials Processing Technology, vol. 249, pp. 479-489, 2017.

[22] P. Bertrand, I. Smurov, and D. Grevey, "Application of near infrared pyrometry for continuous Nd:YAG laser welding of stainless steel," Applied Surface Science, vol. 168, pp. 182-185, 2000.

[23] X. Gao, D. You, and S. Katayama, "Infrared image recognition for seam tracking monitoring during fiber laser welding," Mechatronics, vol. 22, pp. 370-380, 2012.

[24] S. C. A. Alfaro, J. A. R. Vargas, G. C. de Carvalho, and G. G. de Souza, "Characterization of "Humping" in the GTA welding process using infrared images," Journal of Materials Processing Technology, vol. 223, pp. 216-224, 2015.

[25] W. Chen and B. Chin, "Monitoring joint penetration using infrared sensing techniques," Welding Journal, vol. 69, pp. 181$185,1990$.

[26] U. Sreedhar, C. V. Krishnamurthy, K. Balasubramaniam, V. D. Raghupathy, and S. Ravisankar, "Automatic defect identification using thermal image analysis for online weld quality monitoring," Journal of Materials Processing Technology, vol. 212, pp. 1557-1566,2012.

[27] H. C. Wikle, S. Kottilingam, R. H. Zee, and B. A. Chin, "Infrared sensing techniques for penetration depth control of the submerged arc welding process," Journal of Materials Processing Technology, vol. 113, pp. 228-233, 2001.

[28] F. Kong, J. Ma, B. Carlson, and R. Kovacevic, "Real-time monitoring of laser welding of galvanized high strength steel in lap joint configuration," Optics \& Laser Technology, vol. 44, pp. 2186-2196, 2012.

[29] T. Sibillano, D. Rizzi, A. Ancona, S. Saludes-Rodil, J. Rodríguez Nieto, H. Chmelíčková, et al., "Spectroscopic monitoring of penetration depth in CO2 Nd:YAG and fiber laser welding processes," Journal of Materials Processing Technology, vol. 212, pp. 910-916, 2012.

[30] Z. Zhang, E. Kannatey-Asibu, S. Chen, Y. Huang, and Y. Xu, "Online defect detection of Al alloy in arc welding based on feature extraction of arc spectroscopy signal," The International Journal of Advanced Manufacturing Technology, vol. 79, pp. 2067-2077, 2015. 
[31] H. Sebestova, H. Chmelickova, L. Nozka, and J. Moudry, "Non-destructive real time monitoring of the laser welding process," Journal of Materials Engineering and Performance, vol. 21, pp. 764-769, 2012.

[32] T. Ilar, I. Eriksson, J. Powell, and A. Kaplan, "Root humping in laser welding-an investigation based on high speed imaging," Physics Procedia, vol. 39, pp. 27-32, 2012.

[33] I. Eriksson, P. Gren, J. Powell, and A. F. Kaplan, "New high-speed photography technique for observation of fluid flow in laser welding," Optical Engineering, vol. 49, pp. 100503-100503-3, 2010.

[34] I. Eriksson, J. Powell, and A. F. Kaplan, "Melt behavior on the keyhole front during high speed laser welding," Optics and Lasers in Engineering, vol. 51, pp. 735-740, 2013.

[35] C. Li, Y. Shi, Y. Gu, and P. Yuan, "Monitoring weld pool oscillation using reflected laser pattern in gas tungsten arc welding," Journal of Materials Processing Technology, vol. 255, pp. 876-885, 2018.

[36] T. Craeghs, S. Clijsters, E. Yasa, F. Bechmann, S. Berumen, and J.-P. Kruth, "Determination of geometrical factors in Layerwise Laser Melting using optical process monitoring," Optics and Lasers in Engineering, vol. 49, pp. 1440-1446, 2011.

[37] S. Liu, F. Zhang, S. Dong, H. Zhang, and F. Liu, "Characteristics analysis of droplet transfer in laser-MAG hybrid welding process," International Journal of Heat and Mass Transfer, vol. 121, pp. 805-811, 2018.

[38] C. Cai, J. Feng, L. Li, and Y. Chen, "Influence of laser on the droplet behavior in short-circuiting, globular, and spray modes of hybrid fiber laser-MIG welding," Optics \& Laser Technology, vol. 83, pp. 108-118, 2016.

[39] S. Rhee and E. Kannatey-Asibu, "Observation of metal transfer during gas metal arc welding," Welding Research Supplement, vol. 71, pp. 381, 1992.

[40] D. You, X. Gao, and S. Katayama, "Monitoring of high-power laser welding using high-speed photographing and image processing," Mechanical Systems and Signal Processing, vol. 49, pp. 39-52, 2014.

[41] S. Katayama, Y. Kawahito, and M. Mizutani, "Elucidation of laser welding phenomena and factors affecting weld penetration and welding defects," Physics Procedia, vol. 5, pp. 9-17, 2010.

[42] G. Li, Y. Cai, and Y. Wu, "Stability information in plasma image of high-power $\mathrm{CO}_{2}$ laser welding," Optics and Lasers in Engineering, vol. 47, pp. 990-994, 2009.

[43] F. Rémy, S. Sonia, C. Fréderic, and B. Francis, "Study of keyhole behaviour for full penetration Nd-Yag CW laser welding," Journal of Physics D: Applied Physics, vol. 38, p. 1881, 2005.

[44] K. Yousuke, M. Masami, and K. Seiji, "Elucidation of high-power fibre laser welding phenomena of stainless steel and effect of factors on weld geometry," Journal of Physics D: Applied Physics, vol. 40, p. 5854, 2007.

[45] A. Matsunawa, N. Seto, J.-D. Kim, M. Mizutani, and S. Katayama, "Dynamics of keyhole and molten pool in high-power $\mathrm{CO}_{2}$ laser welding," in Advanced High-Power Lasers and Applications, 2000, p. 12.

[46] I. Miyamoto, E. Ohmura, and T. Maede, "Dynamic behavior of plume and keyhole in $\mathrm{CO}_{2}$ laser welding," in International Congress on Applications of Lasers \& Electro-Optics, 1997.

[47] M. Zhang, G. Chen, Y. Zhou, and S. Li, "Direct observation of keyhole characteristics in deep penetration laser welding with a $10 \mathrm{~kW}$ fiber laser," Optics express, vol. 21, pp. 19997-20004, 2013.

[48] A. Sansan, L. Zhen, Z. Nan, and W. Rui, "Blind source separation based on principal component analysis-independent component analysis for acoustic signal during laser welding process," in Digital Manufacturing and Automation (ICDMA), 2010 International Conference on, 2010, pp. 336-339.

[49] Z. Luo, W. Liu, Z. Wang, and S. Ao, "Monitoring of laser welding using source localization and tracking processing by microphone array," The International Journal of Advanced Manufacturing Technology, vol. 86, pp. 21-28, 2016.

[50] L. Grad, J. Grum, I. Polajnar, and J. Marko Slabe, "Feasibility study of acoustic signals for on-line monitoring in short circuit gas metal arc welding," International Journal of Machine Tools and Manufacture, vol. 44, pp. 555-561, 2004.

[51] K. Pal, S. Bhattacharya, and S. K. Pal, "Investigation on arc sound and metal transfer modes for on-line monitoring in pulsed gas metal arc welding," Journal of Materials Processing Technology, vol. 210, pp. 1397-1410, 2010.

[52] A. Sumesh, K. Rameshkumar, K. Mohandas, and R. S. Babu, "Use of machine learning algorithms for weld quality monitoring using acoustic signature," Procedia Computer Science, vol. 50, pp. 316-322, 2015.

[53] C.-J. Lee, J.-D. Kim, and Y.-C. Kim, "Study on monitoring of plasma emission signal in lap welding of Zn coated steel sheet using CO2 laser," International Journal of Precision Engineering and Manufacturing, vol. 16, pp. 495-500, 2015.

[54] G. Hongping and W. W. Duley, "A statistical approach to acoustic monitoring of laser welding," Journal of Physics D: Applied Physics, vol. 29, p. 556, 1996.

[55] A. S. Sun, E. Kannatey-Asibu Jr, W. J. Williams, and M. Gartner, "Time-frequency analysis of laser weld signature," in International Symposium on Optical Science and Technology, 2001, pp. 103-114.

[56] Z. Zhang, H. Chen, Y. Xu, J. Zhong, N. Lv, and S. Chen, "Multisensor-based real-time quality monitoring by means of feature extraction, selection and modeling for Al alloy in arc welding," Mechanical Systems and Signal Processing, vol. 60-61, pp. $151-165,2015$

[57] D. Saini and S. Floyd, "An investigation of gas metal arc welding sound signature for on-line quality control," Welding Research Supplement, vol. 77, pp. 172, 1998.

[58] K. Pal, S. Bhattacharya, and S. K. Pal, "Prediction of metal deposition from arc sound and weld temperature signatures in pulsed MIG welding," The International Journal of Advanced Manufacturing Technology, vol. 45, p. 1113, 2009. 
[59] S. Ao, Z. Luo, M. Feng, and F. Yan, "Simulation and experimental analysis of acoustic signal characteristics in laser welding," The International Journal of Advanced Manufacturing Technology, vol. 81, pp. 277-287, 2015.

[60] D. Farson and R. Kim, "Generation of optical and acoustic emissions in laser weld plumes," Journal of Applied Physics, vol. 85, pp. 1329-1336, 1999.

[61] M. E. Khosroshahi, F. A. pour, M. Hadavi, and M. Mahmoodi, "In situ monitoring the pulse $\mathrm{CO}_{2}$ laser interaction with 316-L stainless steel using acoustical signals and plasma analysis," Applied Surface Science, vol. 256, pp. 7421-7427, 2010.

[62] W. Huang and R. Kovacevic, "Feasibility study of using acoustic signals for online monitoring of the depth of weld in the laser welding of high-strength steels," Proceedings of the Institution of Mechanical Engineers, Part B: Journal of Engineering Manufacture, vol. 223, pp. 343-361, 2009.

[63] W. Huang and R. Kovacevic, "A neural network and multiple regression method for the characterization of the depth of weld penetration in laser welding based on acoustic signatures," Journal of Intelligent Manufacturing, vol. 22, pp. 131-143, 2011.

[64] D. Y. You, X. D. Gao, and S. Katayama, "Review of laser welding monitoring," Science and Technology of Welding and Joining, vol. 19, pp. 181-201, 2014.

[65] M. Bastuck, H.-G. Herrmann, B. Wolter, D. BÖttger, and P.-C. Zinn, "AkuProLas: Acoustic inline process monitoring for laser welding applications," in World Conference on Nondestructive Testing (19): WCNDT 2016, 2016.

[66] A. G. Beattie, "Acoustic emission non-destructive testing of structures using source location techniques," Albuquerque and Livermore, 2013.

[67] M. F. M. Yusof, M. Ishak, and M. F. Ghazali, "Feasibility of using acoustic method in monitoring the penetration status during the Pulse Mode Laser Welding process," IOP Conference Series: Materials Science and Engineering, vol. 238, p. 012006, 2017.

[68] M. F. M. Yusof, M. A. Kamaruzaman, M. Ishak, and M. F. Ghazali, "Porosity detection by analyzing arc sound signal acquired during the welding process of gas pipeline steel," The International Journal of Advanced Manufacturing Technology, vol. 89, pp. 3661-3670, 2017.

[69] M. Čudina, J. Prezelj, and I. Polajnar, "Use of audible sound for on-line monitoring of gas metal arc welding process," Metalurgija, vol. 47, pp. 81-85, 2008.

[70] Y. Wang and P. Zhao, "Noncontact acoustic analysis monitoring of plasma arc welding," International Journal of Pressure Vessels and Piping, vol. 78, pp. 43-47, 2001.

[71] D. Fan, Y. Shi, and M. Ushio, "Investigation of $\mathrm{CO}_{2}$ welding arc sound: Correlation of welding arc sound signal with welding spatter (Physics, Processes, Instruments \& Measurements)," Transactions of JWRI, vol. 30, pp. 29-33, 2001.

[72] J. Horvat, J. Prezelj, I. Polajnar, and M. Čudina, "Monitoring gas metal arc welding process by using audible sound signal," Strojniški vestnik-Journal of Mechanical Engineering, vol. 57, pp. 267-278, 2011.

[73] J. Prezelj and M. Čudina, "Noise as a signal for on-line estimation and monitoring of welding process," ACTA Acustica United with Acustica, vol. 89, pp. 280-286, 2003.

[74] J. Wang, H. Yu, Y. Qian, R. Yang, and S. Chen, "Feature extraction in welding penetration monitoring with arc sound signals," Proceedings of the Institution of Mechanical Engineers, Part B: Journal of Engineering Manufacture, vol. 225, pp. 1683-1691, 2011.

[75] Y. Xiao and G. Den Ouden, "A study of GTA weld pool oscillation," Weld. J., vol. 69, p. 289, 1990.

[76] Q. Wang, C. Yang, and Z. Geng, "Separately excited resonance phenomenon of the weld pool and its application," Welding Research Supplement, vol. 72, pp. 455, 1993.

[77] K. Andersen, G. E. Cook, R. J. Barnett, and A. M. Strauss, "Synchronous weld pool oscillation for monitoring and control," IEEE Transactions on Industry Applications, vol. 33, pp. 464-471, 1997.

[78] N. Lv, Y. Xu, S. Li, X. Yu, and S. Chen, "Automated control of welding penetration based on audio sensing technology," Journal of Materials Processing Technology, vol. 250, pp. 81-98, 2017.

[79] M. G. Drouet and F. Nadeau, "Acoustic measurement of the arc voltage applicable to arc welding and arc furnaces," Journal of Physics E: Scientific Instruments, vol. 15, p. 268, 1982.

[80] L. Grad, J. Prezelj, I. Polajnar, and J. Grum, "Welding process assessment by analyzing on line measured acoustic signals," in 6 th International Conference of Slovenian Society for Nondestructive Testing, 2001, pp. 185-190.

[81] A. E. Wehrmeister, "Weld monitoring with acoustic emission," JOM, vol. 30, pp. 28-30, 1978.

[82] M. G. Droubi, N. H. Faisal, F. Orr, J. A. Steel, and M. El-Shaib, "Acoustic emission method for defect detection and identification in carbon steel welded joints," Journal of Constructional Steel Research, vol. 134, pp. 28-37, 2017.

[83] L. N. Stepanova, S. I. Kabanov, I. S. Ramazanov, E. Y. Lebedev, V. V. Kireenko, A. V. Vonsovskii, et al., "Acoustic-emission testing of multiple-pass welding defects of large-size constructions," Russian Journal of Nondestructive Testing, vol. 51, pp. 540-545, 2015.

[84] Y. Luo, Y. Du, L. Zhu, J. Han, R. Wan, and X. Xie, "Study on the thermo-effect of P-GMAW characterized by structure-borne acoustic emission signals detected in welding on aluminum alloy," Measurement, vol. 92, pp. 200-207, 2016.

[85] Y. Takayama, H. Cho, and T. Matsuo, "Detection of acoustic emission (AE) from Zinc emibrittlement cracking during welding using optical fiber AE monitoring system," Journal of Nondestructive Evaluation, vol. 31, pp. 208-214, 2012.

[86] M. Fidali, "Detection of Welding Process Instabilities Using Acoustic Signals," in Advances in Technical Diagnostics, Cham, 2018, pp. 191-201. 
[87] E. H. Cayo and S. C. A. Alfaro, "A non-intrusive GMA welding process quality monitoring system using acoustic sensing," Sensors, vol. 9, pp. 7150-7166, 2009.

[88] K. Pal and S. K. Pal, "Monitoring of weld penetration using arc acoustics," Materials and Manufacturing Processes, vol. 26, pp. 684-693,2011.

[89] J. Wang, B. Chen, H. Chen, and S. Chen, "Analysis of arc sound characteristics for gas tungsten argon welding," Sensor review, vol. 29, pp. 240-249, 2009.

[90] E. Saad, H. Wang, and R. Kovacevic, "Classification of molten pool modes in variable polarity plasma arc welding based on acoustic signature," Journal of Materials Processing Technology, vol. 174, pp. 127-136, 2006.

[91] Z. Ye, J. Wang, and S. Chen, "Feature selection of arc acoustic signals used for penetration monitoring," in Robotic Welding, Intelligence and Automation, Berlin, Heidelberg, 2011, pp. 203-210.

[92] V. Weerasinghe, J. Kamalu, R. Hibberd, and W. Steen, "Acoustic signals from laser back reflections," Optics \& Laser Technology, vol. 22, pp. 381-386, 1990.

[93] E. T. Smith, "Monitoring laser weld quality using acoustic signals," 1999.

[94] M. Ali, "In-process quality monitoring of laser welds using multi-sensor measurements," The Ohio State University, 1999.

[95] A. P. Dowling and J. Ffowcs Williams, Sound and sources of sound: Horwood, 1983.

[96] L. Li, "A comparative study of ultrasound emission characteristics in laser processing," Applied Surface Science, vol. 186, pp. 604-610, 2002

[97] G. Lewis and R. Dixon, "Plasma monitoring of laser beam welds," Laser, vol. 13, p. 2, 1984.

[98] S. Conesa, S. Palanco, and J. J. Laserna, "Acoustic and optical emission during laser-induced plasma formation," Spectrochimica Acta Part B: Atomic Spectroscopy, vol. 59, pp. 1395-1401, 2004.

[99] D. Farson, A. Ali, and X. Li, "Laser weld penetration monitoring with multiple emission signal measurements," Journal of Laser Applications, vol. 11, pp. 47-53, 1999.

[100] W. W. Duley and Y. L. Mao, "The effect of surface condition on acoustic emission during welding of aluminium with $\mathrm{CO}_{2}$ laser radiation," Journal of Physics D: Applied Physics, vol. 27, p. 1379, 1994.

[101] D. Farson, K. Hillsley, J. Sames, and R. Young, "Frequency-time characteristics of air - borne signals from laser welds," Journal of Laser Applications, vol. 8, pp. 33-42, 1996.

[102] Z. Szymanski, J. Hoffman, and J. Kurzyna, "Plasma plume oscillations during welding of thin metal sheets with a CW CO 2 laser," Journal of Physics D: Applied Physics, vol. 34, p. 189, 2001.

[103] D. Farson, A. Ali, and Y. SANG, "Relationship of optical and acoustic emissions to laser weld penetration," Welding journal, vol. 77, pp. 142, 1998.

[104] C. F. Boulton, "Fatigue life predictions of welded specimens containing lack of penetration defects at ambient and elevated temperatures," International Journal of Pressure Vessels and Piping, vol. 4, pp. 171-195, 1976.

[105] F. Lawrence and W. Munse, "Fatigue crack propagation in butt welds containing joint penetration defects," Welding Journal, vol. 52, 1973.

[106] P. J. Singh, D. R. G. Achar, B. Guha, and H. Nordberg, "Influence of weld geometry and process on fatigue crack growth characteristics of AISI 304L cruciform joints containing lack of penetration defects," Science and Technology of Welding and Joining, vol. 7, pp. 306-312, 2002. 